\title{
LITOS DISCOIDALES TEMPRANOS EN CONTEXTOS DE PATAGONIA
}

\author{
DONALD JACKSON S. Y CÉSAR MÉNDEZ M.
}

\begin{abstract}
RESUMEN
En algunos sitios Paleoindios del extremo austral de Sudamérica (Patagonia) se ha registrado litos discoidales pulidos como artefactos diagnósticos de las primeras ocupaciones humanas. Estas piezas han sido halladas en contextos residenciales al interior de reparos rocosos asociadas a las tradicionales puntas de proyectil "cola de pescado" y fauna extinta. En este trabajo se discuten conocidas y nuevas evidencias de hallazgos de litos discoidales haciendo hincapié en la dimensión contextual de los hallazgos. Se discute su eventual función simbólica y relación con otros litos análogos procedentes de contextos costeros del Pacífico de Sudamérica. Se sugieren algunas conexiones culturales entre las poblaciones tempranas que ingresaron al continente durante la transición Pleistoceno-Holoceno.
\end{abstract}

PALABRAS CLAVES: litos discoidales, Paleoindio, transición Pleistoceno-Holoceno, Sudamérica.

\section{EARLY DISCOIDAL STONES IN PALEOINDIAN CONTEXTS OF SOUTH AMERICA}

\begin{abstract}
Polished discoidal stones are diagnostic artefacts associated to fish tail projectile points and extinct fauna in a number of Paleoindian cave sites in southernmost South America (Patagonia). In this paper we examine the contextual associations of currently known and hitherto unreported finds of discoidal stones and their relations to similar artefacts found along the Pacific coast of South America. We also discuss their potential symbolic functions and explore the suggestion that they signal cultural relations between the human groups that peopled the continent during the Pleistocene-Holocene transition.
\end{abstract}

KEY WORDS: discoidal stones, Paleoindian, Pleistocene-Holocene transition, South America.

- Departamento de Antropología, Facultad de Ciencias Sociales, Universidad de Chile. Ignacio Carrera Pinto 1045, Ñuñoa, Santiago de Chile, E-mail: djackson@uchile.cl, cmendezm@uchile.cl 


\section{INTRODUCCIÓN}

Los primeros descubrimientos de sitios paleoindios en el extremo austral del continente ocurrieron hacia mediados de la década del 30' en Cueva Fell y Pali Aike (Bird 1938, 1993). En ambas cuevas se pudo constatar largas secuencias que permitieron formar la base secuencial de cinco períodos culturales que ha servido como ordenamiento de las ocupaciones humanas originarias de Patagonia (Bird 1993).

En los niveles más profundos de ambos asentamientos se registró por primera vez, en estratigrafía, asociaciones certeras entre instrumentos líticos de clara factura humana, fogones, restos de fauna extinta y otras evidencias culturales datadas hacia la transición Pleistoceno-Holoceno (Bird 1993). A estos hallazgos se sumaron tempranamente las investigaciones en la cueva de Los Toldos, con similares contextos asociados (Menghin 1952).

Entre las evidencias culturales de Cueva Fell, Pali Aike y Los Toldos se registraron cuatro litos discoidales (figura 1). Fueron descritos y discutidos por Bird (1970, 1993), destacándose su asociación a puntas "cola de pescado" y fauna extinta, en el marco de contextos de filiación paleoindia. Bird señaló que: La comparación de los cuatro especímenes no ayuda para resolver el enigma de sus funciones. Las marcadas variaciones en tamaño y naturaleza del material, dejan solamente la forma y la falta de abrasión por uso obvio, como características compartidas. Hasta que otras puedan encontrarse, pasarán muchos años antes que esté disponible para estudio una amplia serie (1993:172, original en Bird 1970).

Treinta y siete años después, la evidencia de litos discoidales procedentes de contextos arqueológicos no ha aumentado significativamente. Sin embargo, algunos nuevos hallazgos ponen de manifiesto, por una parte, la reiteración de nuevos litos en contextos Paleoindios -denotando su carácter de indicador cultural-y por otra, su singular presencia en contextos habitacionales intensamente ocupados. Asimismo, la presencia de litos discoidales en otros contextos tempranos de la costa Pacífica de Sudamérica, sugiere algún vínculo o sustrato cultural común entre estas poblaciones que ingresaron tempranamente al continente durante la transición Pleistoceno-Holoceno.

\section{LOS SITIOS CON LITOS DISCOIDALES Y SUS CONTEXTOS}

Las evidencias de litos discoidales en el extremo sur de Sudamérica se reducen a sólo 11 piezas. Seis se encuentran en estratigrafía en sitios con contextos bien datados, mientras que las restantes corresponden a dos hallazgos superficiales y a un contexto estratigráfico poco definible (figuras 1 y 2).

Las primeras evidencias de litos discoidales provienen de Cueva Fell (figura 1a) (Bird 1938, 1993). Se trata de una cueva situada en la ribera sur del río Chico o Ciaike, en la región volcánica oriental de Pali Aike, Patagonia meridional. En el nivel inferior de esta cueva se identifica un primer momento ocupacional (Unidad Cultural Fell I) con un amplio registro arqueológico que incluye la presencia de fauna extinta (Hippidion saldiasi), cánidos (Dusicyon avus) y fauna moderna (Lama guanicoe), asociada a fogones en cubeta $e$ instrumentos de hueso y líticos. Entre los últimos se incluyen lascas, raspadores, raederas, cuchillos, puntas de proyectil y tres litos discoidales ${ }^{1}$. Este contexto ha sido datado hacia los 11.000 años AP (Emperaire et al. 1963, Bird 1938, 1988, Massone 1981, Caviglia 1986, Massone y Prieto 2004, Alberdi y Prieto 2000).

En la misma región y a sólo unos $40 \mathrm{~km}$ hacia el este de Cueva Fell, se sitúa en una formación de lava, la cueva de Pali Aike, también excavada por Bird (1988) en similares fechas. En los niveles inferiores de esta cueva, se registró un componente muy similar al detectado en Cueva Fell, con presencia de caballo nativo (Hippidion saldiasi) asociado a dos fogones en cubeta $e$ instrumental lítico, entre los que destaca un fragmento basal de punta "cola de pescado" y un lito discoidal con algunas fracturas (figura 1b). Este contexto fue datado hacia los 8.600 años AP, fecha tardía para un contexto paleoindio y cuestionada, ya que se trata de una muestra de huesos de distintos animales y por tanto, muy promediada (Bird 1938, 1988, Massone 1981, Massone y Prieto 2004, Alberdi y Prieto 2000).

1 Para Cueva Fell se han descrito dos litos discoidales (Bird 1988). Posteriores excavaciones realizadas por John Fell (1959) en los niveles inferiores de la cueva permitieron registrar una nueva pieza, la que se encuentra depositada en las colecciones del Instituto de la Patagonia, Universidad de Magallanes, Punta Arenas. 

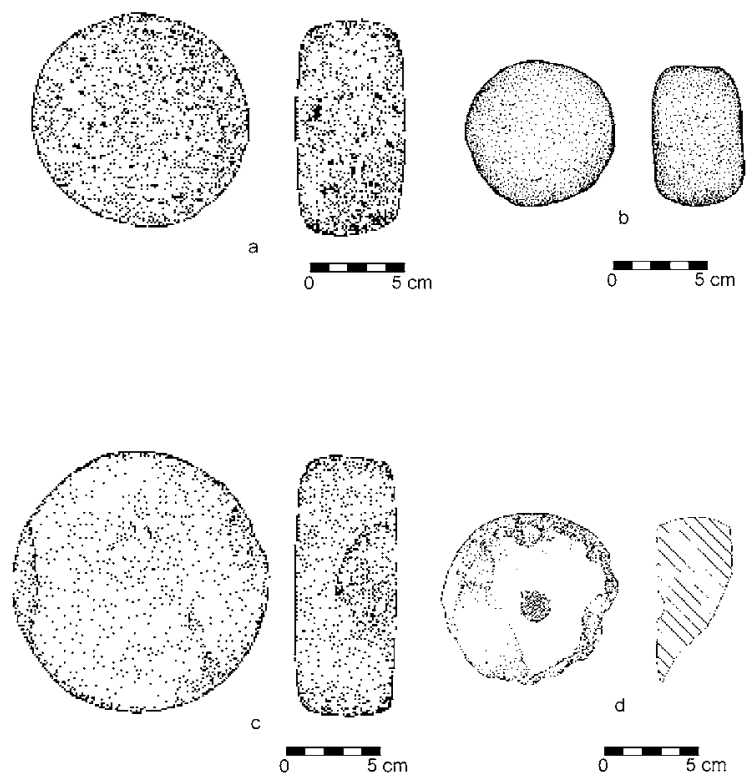

Fig. 1. Litos discoidales, a. Cueva Fell, b. Pali Aike, c. Los Toldos Cueva 2 y d. Cerro El Sombrero.

En Patagonia centro-meridional, al sur del río Deseado, en la provincia de Santa Cruz, dos cuevas en la localidad de Los Toldos ( 2 y 3), muestran una ocupación humana llamada "Industria del Nivel 11" datada hacia los 12.600 años AP en la cueva 3. Inmediatamente sobre esta ocupación, en los niveles 10 y 9 (este último datado en su cima hacia los 8.750 años AP) se definió un complejo cultural denominado Toldense, análogo a la Unidad Cultural Fell I. Ambas dataciones, no obstante, deben ser consideradas con reserva, en vista de las muestras seleccionadas, sus contextos informativos (estratigrafía y asociaciones) y necesidad de reevaluación (Borrero 1999). El registro arqueológico en este sitio muestra la presencia de raederas, raspadores y piezas bifaciales de variada morfología, desechos líticos y la presencia de un fragmento de lito discoidal. Por su parte, en la cueva 2 , en niveles asignables también al Toldense, se registró fauna actual y caballo nativo (Hippidion saldiasi), dos fragmentos de puntas "cola de pescado" y un lito discoidal (figura 1c), en asociación a otros instrumentos y desechos líticos (Menghin 1952, Cardich et al. 1973, Mengoni 1980, Alberdi y Prado 2004).

Al norte de la Patagonia oriental, en la región pampeana de la provincia de Buenos Aires, en el sitio denominado Cerro El Sombrero, localizado en
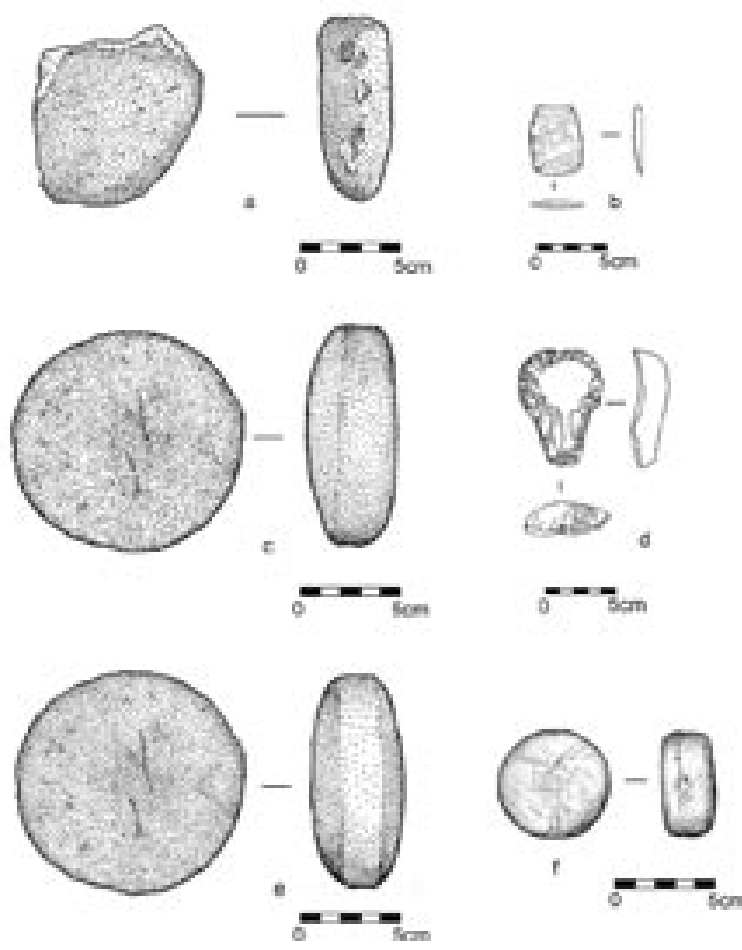

Fig. 2. Litos discoidales y asociación a otros materiales: a. lito discoidal fracturado (Baño Nuevo 1), b. punta de proyectil (Baño Nuevo 1), c. lito discoidal (Appeleg 1), d. raspador (Appeleg 1), e. lito discoidal (río Huemules) y f. lito discoidal (Río Ibáñez 18).

la sierra de Tandilla, se han definido dos unidades de asentamiento: El Abrigo 1 y La Cima. Allí se registraron evidencias superficiales y estratigráficas de una ocupación paleoindia identificada por la presencia de núcleos, desechos de talla, lascas retocadas, bifaciales fragmentados, cuchillos bifaciales, raspadores, raclettes, limaces y numerosas puntas "cola de pescado", elaboradas esencialmente en ortocuarzita de excelente calidad. Junto a estos instrumentos se registró un lito discoidal en arenisca (figura 1d), así como restos de ocre. El sector de El Abrigo ha sido fechado hacia los 10.500 años AP (Flegenheimer y Zárate 1989, Flegenheimer 2003, Mazzia et al. 2005), fecha parámetro para ambos contextos (bajo reparo y cielo abierto).

En Patagonia central, en la Región de Aisén, en una cueva denominada Baño Nuevo 1 (Alto Nirehuao), se ha registrado una secuencia con varios eventos ocupacionales a lo largo del Holoceno. En la capa 3 se identificó una ocupación asociada a 
fogones, restos de 10 disposiciones mortuorias (al menos tres neonatos y dos adultos, un hombre y una mujer) $e$ instrumental lítico que incluye puntas apedunculadas (figura $2 \mathrm{~b}$ ), raspadores, cuchillos, lascas y láminas con huellas de uso, desechos de talla y un gancho de estólica elaborado en hueso. Esta ocupación se asocia con restos de fauna moderna (Lama guanicoe y cánidos), integrando un componente temprano datado entre los 9.500 y 8.000 años AP (Mena y Reyes 1998, Trejo y Jackson 1998, Mena et al. 2000, Velásquez y Mena 2006). Un lito discoidal fragmentado (figura 2a) se registró en la base de la capa 3 (unidad 1B) en proximidad a un punzón óseo directamente datado hacia los 7.500 años AP (Velásquez y Mena 2006). No obstante, no debe excluirse que los procesos de entierro de los cuerpos humanos pudieran remover la pieza desde su posición original. En este sentido no debiera descartarse una eventual cronología más temprana.

Otros hallazgos en la misma región poseen contextos crono-culturales menos claros. Este es el caso del sitio localizado en el Juncal Alto en Río Ibáñez (RI-18), un alero con pictografías y tres estratos ocupacionales (Berquist et al. 1983). El estrato I muestra evidencias análogas a la Unidad Cultural Tardía Fell V, en el estrato II, un entierro masculino adulto datado en $410 \pm 40$ años AP y en el más profundo $(\sim 80 \mathrm{~cm})$, se registró la presencia de un lito discoidal completo elaborado en arenisca (figura 2f), asociado a desechos de talla y un núcleo de basalto, desconociéndose otros datos del contexto de recuperación (Berqvist et al. 1983, Mena 2000, Reyes 2002).

También se ha constatado otros hallazgos en superficie, uno de ellos aislado en el margen norte del río Huemules, sector del cerro Galera, también en la Región de Aisén. Se trata de un lito discoidal completo (figura $2 e$ ) con superficies biconvexas y borde perimetral ligeramente recto. En esta misma localidad se registró una punta "cola de pescado" en proceso de elaboración (Bate 1982), lo que podría estar sugiriendo la presencia de algún pequeño campamento a cielo abierto o "paradero" de cazadores paleoindios en las proximidades de la localidad.

Asimismo, recientes investigaciones en el alto río Cisnes $\left(44^{\circ} \mathrm{S}\right)$, también en Aisén, han permitido registrar en un extenso asentamiento multicomponente designado como Appeleg 1 (CIS
009), en un sector de dunas en deflación. Dominan diversas categorías líticas diagnósticas de la unidad cultural tardía, como puntas de proyectil y raspadores frontales. Aún así, se pudo identificar puntas de proyectil triangulares apedunculadas, similares tipológicamente a aquellas registradas en el componente temprano de Baño Nuevo 1. En un área de concentración de evidencias superficiales (unidad 37) se registró un lito discoidal completo; mientras que en otra concentración (unidad 1) se observó un gran raspador enmangable (figura $2 \mathrm{~d}$ ) de notable similitud a los identificados en el nivel temprano de Cueva Fell (Méndez et al. 2007). Este raspador presenta intensa patinación $e$ indicios de haber sido reactivado con posterioridad.

Las evidencias de litos discoidales, con la excepción de Río Ibáñez 18 y Appeleg 1, muestran una clara asociación estratigráfica con contextos tempranos, definidos regionalmente como Unidad Cultural Fell 1 (Bird 1938, 1993, Massone 1981). La presencia de puntas de proyectil "cola de pescado" en Cueva Fell, Pali Aike, Cueva 2 de Los Toldos y en Cerro El Sombrero constituye uno de los indicadores diagnósticos para los primeros poblamientos de Sudamérica; como también lo es la presencia de fogones en cubeta en los dos primeros y su clara asociación con fauna extinta en varios de los sitios mencionados. Los atributos descritos confirmarían, por una parte, el carácter temprano de estas ocupaciones, y sumados a los litos discoidales permitirían robustecer el conjunto de indicadores crono-culturales. Por otra parte, la Cueva 3 de Los Toldos y Baño Nuevo 1 presentan a los litos discoidales en asociación a puntas de proyectil apedunculadas triangulares, asignables a lo que se ha denominado Toldense, sin fauna extinta asociada en el caso del segundo.

Las dataciones radiocarbónicas de los contextos discutidos (tabla 1) muestran una cronología asignable a la transición Pleistoceno Holoceno para las ocupaciones con litos discoidales cuando están asociados a fauna extinta y puntas "cola de pescado", mientras que exhiben una cronología más tardía en su asociación a puntas de proyectil triangulares, de contextos "Toldense" de Patagonia central. De considerarse los litos discoidales como indicadores diagnósticos válidos, ello sugeriría que existirían elementos de un sustrato estilístico común entre las poblaciones de la transición Pleistoceno 
Tabla 1. Dataciones (sin calibrar) de los contextos con litos discoidales.

\begin{tabular}{|c|c|c|c|}
\hline Sitios & Dataciones (años AP.) & Muestra & Referencias \\
\hline Cueva Fell & $\begin{array}{l}11.000 \quad \pm 170 \\
10.720 \quad \pm 300\end{array}$ & $\begin{array}{l}\text { Carbón } \\
\text { Carbón }\end{array}$ & Bird 1993 \\
\hline Cueva Pali Aike & $8.639 \pm 450$ & Hueso & Bird 1993 \\
\hline $\begin{array}{l}\text { Cueva } 3 \text { Los Toldos } \\
\text { Nivel } 11 \\
\text { Techo nivel } 9\end{array}$ & $\begin{aligned} 12.600 & \pm 600 \\
8.730 & \pm 480\end{aligned}$ & $\begin{array}{l}\text { Carbón } \\
\text { Carbón }\end{array}$ & Cardich et al. 1973 \\
\hline Cerro El Sombrero & $\begin{array}{ll}10.725 & \pm 90 \\
10.675 & \pm 110 \\
10.480 & \pm 70 \\
10.270 & \pm 85\end{array}$ & $\begin{array}{l}\text { Carbón } \\
\text { Carbón } \\
\text { Carbón } \\
\text { Carbón }\end{array}$ & Flegenheimer 2003* \\
\hline $\begin{array}{l}\text { Baño Nuevo } 1 \\
\text { Componente Temprano }\end{array}$ & $\begin{array}{l}18 \text { dataciones entre los } \\
9.530 \pm 25 \text { y } 8.530 \pm 160\end{array}$ & $\begin{array}{l}\text { Carbón } \\
\text { Hueso } \\
\text { Coprolito }\end{array}$ & Velásquez y Mena 2006 \\
\hline $\begin{array}{l}\text { Componente Medio } \\
\text { "datación asociada" }\end{array}$ & $\begin{array}{c}6 \text { dataciones entre los } \\
7.990 \pm 20 \text { y } 5.095 \pm 15 \\
\rightarrow 7.500 \pm 20\end{array}$ & Punzón & Velásquez y Mena 2006 \\
\hline
\end{tabular}

* Una fecha más tardía ( 8.000 AP) se considera contaminada y no se recomienda como válida.

Holoceno (fechas pre $10.000 \mathrm{AP}$ ) y aquellas datadas ya entrado el Holoceno Temprano, al menos en Patagonia central.

Las evidencias de litos discoidales en los sitios referidos estarían presentes únicamente en contextos de carácter residencial y particularmente bajo reparo rocoso (Cueva Fell, Pali Aike, Los Toldos, Cerro El Sombrero y Baño Nuevo) tipo de asentamiento característico del proceso inicial de poblamiento de Patagonia (Borrero y Franco 1997, Borrero 1999, Massone 1999). Estas piezas se han observado en ocupaciones probablemente intensas y reiteradas como lo sugieren los densos depósitos de actividad doméstica, que incluyen fogones en la mayoría de los sitios (aunque en Cerro El Sombrero su presencia está atestiguada sólo por partículas de carbón). Las evidencias se hallan asociadas -frecuentemente- a una alta diversidad funcional de instrumentos líticos, tanto elaborados, como usados, reactivados y descartados localmente. Entre ellos destacan puntas de proyectil, cuchillos, raederas, raspadores y múltiples subproductos del proceso de talla. Otras evidencias significativas de actividades domésticas diversificadas son tanto la presencia de instrumentos en hueso (Cueva Fell, Pali Aike y Baño Nuevo 1), como pigmentos (Los Toldos, Baño Nuevo 1 y Cerro El Sombrero). Los sitios observados presentan invariablemente restos de fauna actual (Cueva Fell, Pali Aike, Los Toldos, Baño Nuevo) y también taxones extintos (Cueva
Fell, Pali Aike y Los Toldos), característica de la amplitud selectiva durante el poblamiento inicial (Borrero y Franco 1997). El único sitio con una función particular que escapa a la norma es Baño Nuevo 1, donde se ubicaron entierros humanos reiteradamente depositados. No obstante, la asociación funcional entre el lito discoidal descrito y alguno(s) de los entierros no es del todo certera y merece mayor discusión.

Los hallazgos superficiales de litos discoidales deben ser considerados con mucha cautela en función de los procesos de formación que afectaron su descontextualización. El caso Río Huemules, donde se registró una pieza aislada, resulta poco significativo por sí solo. No obstante, la presencia en la misma localidad de una punta de proyectil "cola de pescado" podría estar sugiriendo algún sitio a cielo abierto con mayor cantidad de evidencias en las cercanías. En el caso de Appeleg 1 se trataría de un yacimiento erosionado y en situación de evidente palimpsesto con materiales de cronología tardía. Sin embargo, al igual que el sitio anterior de detectarse una ocupación más clara, ésta también sería a cielo abierto; modalidad hasta ahora no detectada en Patagonia. Estas efímeras evidencias, junto con el lito discoidal de Río Ibáñez 18, en contexto estratigráfico poco definible, deben ser reevaluadas a la luz de nuevos antecedentes contextuales y cronológicos, diseñándose métodos para abordar el problema particular. 


\section{LOS LITOS DISCOIDALES: CARACTERÍSTICAS FORMALES Y FUNCIÓN}

Morfológicamente, los litos son discoidales con sus caras de superficie convexas, siendo algo más espesas en su centro y disminuyendo levemente hacia su periferia. Los bordes perimetrales son rectos y ligeramente convexos en su inflexión con las caras. Sólo un lito (Cerro El Sombrero) muestra al centro de una de sus caras, una pequeña oquedad la cual ha sido grabada en forma de reticulado.

Hallazgos superficiales en Uruguay atribuidos a grupos paleoindios muestran lo que se ha descrito como "manos" con oquedades en el centro de las piezas (Taddei 1987), no obstante su morfología (contorno general y especialmente sus secciones) sugiere que se trataría más bien de litos discoidales similares al encontrado en Cerro El Sombrero, aunque con la variación referida en su centro. Hallazgos estratigráficos de puntas denominadas "Pay Paso" en contextos paleoindios bien datados (Suárez 2003, Suárez y López 2003) y similares a las registradas en superficie junto a estos litos discoidales tienden a apoyar una cronología temprana para estos hallazgos, así como la idea que serían litos y no la función originalmente asignada.

Las materias primas (tabla 2) utilizadas en la confección de las piezas han sido lavas (máfica y félsica), tobas, argilita, arenisca y granito. La gran mayoría de ellas son rocas locales, siendo las más utilizadas las areniscas y lavas. Las características de estas rocas sugieren que han sido seleccionadas por su textura y tamaño de grano (faneríticas), lo que debió facilitar el proceso de elaboración. Este proceso probablemente consideró primero la percusión (trituramiento) y luego por abrasión y desgaste, para dar el acabado.

Es posible que para su elaboración se seleccionaran trozos de rocas naturales cuya forma se adecuara a los productos discoidales. Sólo dos piezas de las analizadas se encuentran en proceso de elaboración. La primera, de Cueva Fell, muestra desgaste sólo en una de sus caras y parcialmente en los bordes, sugiriendo la utilización de una matriz ovoidal de lava. La otra, procedente de Baño Nuevo 1 , atestigua huellas de desgaste en una cara y en la otra trituramiento y abrasión inicial. Adicionalmente, la segunda se encuentra fracturada. El acabado final de las piezas es muy esmerado en términos de la proporcionalidad de sus dimensiones, así como la regularidad de sus caras y bordes.

Respecto a sus dimensiones (tabla 2), los litos discoidales muestran una interesante variabilidad de tamaños generales (diámetros), no así en sus espesores. El más grande corresponde al hallazgo de Cueva 2 de Los Toldos (14.8-14.95-5.3 cm), mientras que el más pequeño es el registrado en Río Ibáñez 18 (5.73-5.44-2.74 cm). Preliminarmente, no se observaría estandarización en dimensiones de los diámetros, aunque la muestra es muy pequeña para tener mayor confiabilidad. Aún así, el hallazgo de Río Ibáñez 18, además de ser el lito discoidal más

Tabla 2. Características de los litos discoidales por sitio. Dimensiones en cm, *diámetro 1, diámetro 2, espesor;

**medidas estimadas, D: diámetro, esp: espesor, índice de regularidad entre D.1 y D.2 para las piezas completas.

\begin{tabular}{|c|c|c|c|c|c|c|c|}
\hline \multirow[t]{2}{*}{ Sitios } & Materia prima & \multicolumn{3}{|c|}{ Dimensiones* } & \multirow[t]{2}{*}{ Conservación } & \multirow[t]{2}{*}{$\begin{array}{c}\text { Índice } \\
\text { regularidad }\end{array}$} & \multirow[t]{2}{*}{ Referencias } \\
\hline & & D. 1 & D. 2 & Esp. & & & \\
\hline Cueva Fell & lava máfica & 12,35 & 12,2 & 6,1 & completo & 1,01 & Bird 1988 \\
\hline Cueva Fell & lava félsica & 8,5 & 8,3 & 4,2 & completo & 1,02 & Bird 1988 \\
\hline Cueva Fell & lava & & & & completo & & Fell 1959 \\
\hline Pali Aike & toba & 8,3 & 7,9 & 5,2 & fracturado & & Bird 1988 \\
\hline Los Toldos C. 2 & argilita & 14,8 & 14,95 & 5,3 & completo & 0,99 & Menghin 1952 \\
\hline Los Toldos C. 3 & $\dot{¿} ?$ & 9,3 & 5 & 5,9 & fracturado & & Cardich et al. 1973 \\
\hline Co. El Sombrero & arenisca & & 9,8 & 4,1 & fracturado & & Flegenheimer y Zárate 1989 \\
\hline Baño Nuevo 1 & arenisca & 9,77 & 7,91 & 3,77 & fracturado & & Mena 2004 \\
\hline Río Ibáñez 18 & arenisca & 5,73 & 5,44 & 2,74 & completo & 1,05 & Mena 2000 \\
\hline Río Huemules & $\dot{i} ?$ & & & & completo & & Bate 1982 \\
\hline Appeleg 1 & granito & 10,96 & 10,51 & 4,25 & completo & 1,04 & Méndez et al. 2007 \\
\hline Media & & 9,96 & 9,11 & 4,62 & & & \\
\hline ds & & 2,76 & 3,16 & 1,09 & & & \\
\hline
\end{tabular}


pequeño en un contexto muy dudoso, se escapa bastante de la media.

La primera impresión interpretativa de los litos discoidales sugeriría su uso como manos de moler o sobadores, aun cuando en Patagonia y Pampa el uso de implementos de molienda es muy escaso durante el poblamiento inicial. Sin embargo, la observación microscópica de las piezas de Cueva Fell, no atestiguó ningún indicio de residuos o huellas que así lo demostraran (Bird 1970, 1993). Adicionalmente, un examen bajo lupa (80x) de las piezas de la Región de Aisén (Baño Nuevo, Appeleg 1 y Río Ibáñez 18), tampoco mostró patrones de huellas (desgaste y/o estrías) que pudieran ser atribuidas a la acción de molienda o su uso como sobadores de cuero (Adams 1988, 1999). El único caso especial es el lito discoidal de Río Ibáñez 18 que mostraba restos de pigmento rojo en toda su superficie, sin indicios que sugirieran que esta pieza se hubiera usada para esparcirlos, sino más bien que se cubrió con ellos.

\section{DISCUSIÓN Y CONCLUSIONES}

Los escasos -aunque significativos- antecedentes contextuales de litos discoidales paleoindios del extremo austral del continente permiten plantear preliminarmente que estas singulares piezas líticas debieron tener una función de carácter simbólico. La diversidad de materias primas empleadas y sus características petrográficas, así como sus diversos tamaños, la ausencia de huellas y residuos de uso, sugieren que los litos discoidales no tienen una función utilitaria de carácter doméstico (p.e. molienda de pigmentos o vegetales). Por el contrario, las características descritas suponen que se priorizaron sus rasgos formales (dimensiones, regularidad y proporcionalidad, tabla 2) por sobre sus características de materia prima y tamaño, lo que sugiere más una función de carácter simbólico, que meramente utilitaria.

Los contextos estratigráficos estarían mostrando que los litos discoidales se encuentran en sitios habitacionales con una intensa actividad doméstica, especialmente bajo reparos rocosos con alta redundancia ocupacional. Más allá de cualquier duda razonable, estos sitios habitacionales debieron albergar unidades familiares integradas por distintos segmentos etáreos y de género.
Otros asentamientos contemporáneos del extremo austral como Cueva del Medio (Nami 1987) y Cueva de Laguna Sofía 1 (Prieto 1991) en Última Esperanza, Piedra Museo en la cuenca del río Deseado (Miotti 1996), Tres Arroyos 1 en la estepa norte de Tierra del Fuego (Massone 1987, 2004) o cueva Los Pinos en la pampa argentina (Valverde 2006) -entre otros-, muestran campamentos habitacionales aparentemente de corta estadía, con escasos depósitos y limitado registro instrumental, independiente de su redundancia ocupacional. En estos contextos no se ha registrado litos discoidales, lo que podría relacionarse a lo breve de sus lapsos ocupacionales.

Lo anterior estaría mostrando la recurrencia de los litos discoidales en sitios habitacionales de relativa mayor permanencia (a excepción tal vez de Cueva Pali Aike), en donde una amplia diversidad de actividades domésticas se llevó a cabo por conjuntos humanos más grandes que, por ejemplo, por grupos de tareas puntuales. La probable redundancia de estas ocupaciones sugiere la selección de espacios privilegiados de habitación como cuevas y aleros rocosos de particulares características, que incluso pueden ser visualizados como hitos geográficos de connotación en el paisaje cultural.

En estos singulares espacios la práctica de ritos pudo estar presente, aunque no necesariamente escindida de otros aspectos domésticos. El denominador común en estos contextos habitacionales, más que la presencia de un segmento social en particular, fue la coexistencia de grupo en su totalidad. En este sentido, los litos discoidales pudieron tener una significación particular en la interacción y reproducción social de las unidades grupales, eligiéndose para ello espacios únicos -domésticos- con lapsos de ocupación más prolongados y por tanto, propicios para el desarrollo de prácticas rituales. Ello también probablemente incidió en la redundancia de estos campamentos.

La unicidad de estas piezas y su baja frecuencia en los contextos referidos aboga en favor de un carácter excepcional, probablemente vinculado a actividades esporádicas. En algunos casos como en Baño Nuevo 1, Los Toldos y Cerro El Sombrero, se asocian a la presencia de pigmentos, que son comúnmente usados en ritos ceremoniales, si bien no de forma excluyente. No es menos significativa la presencia de litos discoidales fracturados al menos 
en cuatro de los sitios. En la cueva 2 de Los Toldos, esto es particularmente claro. La pieza muestra una fractura con huellas de impacto que indican su clara intencionalidad, sugiriendo que ha sido ritualmente "matada", significando probablemente el término de su función simbólica, al igual que lo que sucede con manos de moler y otros instrumentos al término de su vida útil (Deller y Ellis 2001, Jackson 2000). Difícilmente podremos saber con exactitud las funciones de los litos discoidales, no obstante, sabemos que lo simbólico está fuertemente arraigado a la identidad, al fortalecimiento de las relaciones sociales y esencialmente al sistema de reproducción social.

Llama la atención, finalmente, que otras poblaciones que ocuparon la costa del Pacífico de Sudamérica de forma relativamente contemporánea, como el Complejo Cultural Huentelauquén ( 11.000 a 8.500 AP) en el norte y centro de Chile (Llagostera 1979, Llagostera et al. 2000, Jackson et al. 1999, Jackson y Méndez 2005) o el Complejo Las Vegas (10.000 a 6.000 AP) en la Península de
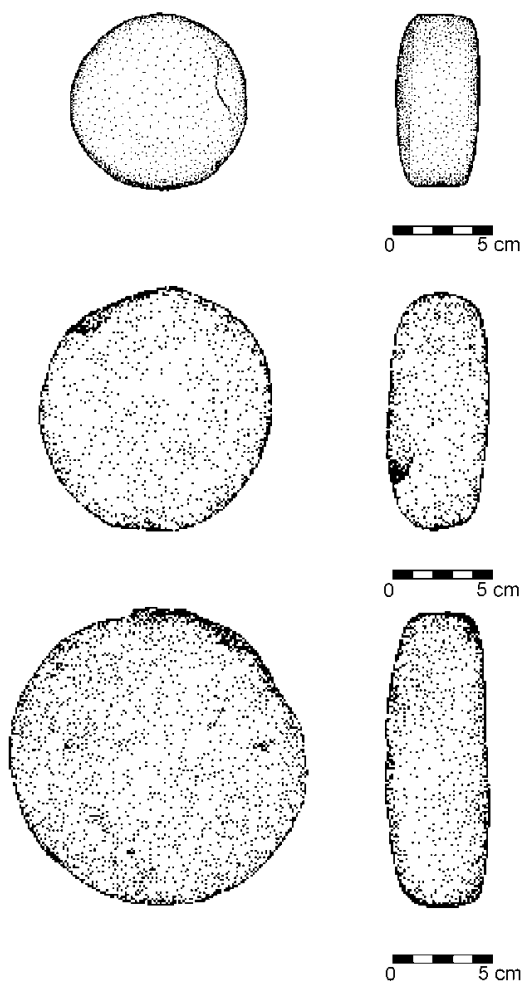

Fig. 3. Litos discoidales del Complejo Cultural Huentelauquén, costa del Norte y Centro de Chile.
Santa Elena de Ecuador (Stothert 1988, Stothert y Quilter 1991), expongan contextos con litos discoidales (figura 3) entre otras formas geométricas. En cuanto a las materias primas seleccionadas dominan las areniscas, aunque no de forma definitiva y en términos formales, muestran una gran regularidad, simetría y secciones espesas muy similares a los litos discoidales de Patagonia. Éstos se presentan sin asociación a fauna extinta, pero están datados hacia la transición Pleistoceno-Holoceno. Al igual que los examinados aquí, análisis de trazas de uso en sus caras resultan negativos para evidencias de molienda, lo que permite descartar hipótesis utilitarias como su propósito de confección. Las similitudes preliminarmente esbozadas permitirían sugerir alguna conexión o sustrato cultural común entre estas poblaciones tempranas que ingresaron a Sudamérica.

\section{AGRADECIMIENTOS}

Agradecemos a Nora Flegenheimer (Museo Municipal de Ciencias Naturales de Necochea, Argentina) por facilitar datos inéditos del lito discoidal de Cerro El Sombrero, a Flavia Morello (Instituto de la Patagonia, Universidad de Magallanes) quien nos facilitó la información de un tercer lito discoidal de Cueva Fell y a Francisco Mena (Museo Chileno de Arte Precolombino) quien gentilmente facilitó la pieza de Baño Nuevo para su estudio. La información del área de río Cisnes (sitio Appeleg 1) fue obtenida por medio del proyecto FONDECYT 1050139, dirigido por Omar Reyes. Agradecemos también a Luis Borrero (Instituto Multidisciplinario de Historia y Ciencias Humanas, Buenos Aires) por los sinceros y pertinentes comentarios al manuscrito y a Ismael Martínez por la ayuda en las ilustraciones.

\section{BIBLIOGRAFÍA}

ADAMS, J. 1988. Use-wear analysis of handstone to gring corn an process hide. Journal of Field Archaeology 15:307-325.

ADAMS, J. 1999. Refocusing the role of food-grinding tools as correlates for subsistence strategies in the U.S. Southwest. American Antiquity 64:475-498.

ALBERDI, M. y A. PRIETO. 2000. Restos de Hippidion saldiasi en las cuevas de la Patagonia Chilena. Anales del Instituto de la Patagonia 28:147-171. 
ALBERDI, M. y J. PRADO. 2004. Caballos fósiles de América del Sur; Una Historia de tres millones de años. Universidad Nacional del Centro de la Provincia de Buenos Aires, INCUAPA, Serie Monográfica 3.

BATE, L. 1982. Orígenes de la Comunidad Primitiva en Patagonia. Serie Monográfica Cuicuilco, Escuela Nacional de Antropología e Historia, Ciudad de México.

BERQVIST, N., V. BUSTOS y N. SANDOVAL.1983. Investigaciones arqueológicas en la comuna de Río Ibáñez, XI Región. Universidad de Concepción. Concepción. MS.

BIRD, J. 1938. Antiquity and migrations of the early inhabitants of Patagonia. The Geographical Review 28:250-275.

BIRD, J. 1970. Paleoindian Discoidal Stones from South America. American Antiquity 35:205-208.

BIRD, J. 1988. Travels and Archaeology in South Chile. (Hyslop, J. Ed.), Iowa University Press, Iowa.

BIRD, J. 1993. Viajes y arqueología en Chile Austral, editado por J. Hyslop. Ediciones de la Universidad de Magallanes, Punta Arenas.

BORRERO, L. 1999. The prehistoric exploration and colonization of Fuego-Patagonia. Journal of World Prehistory 13:321-355.

BORRERO, L. y N. FRANCO. 1997. Early Patagonian HunterGatherers: Subsistence and Technology. Journal of Anthropological Research 53:219-239.

CARDICH, A., L. CARDICH y A. HAJDUK. 1973. Secuencia arqueológica y cronología radiocarbónica de la Cueva 3 de Los Toldos (Santa Cruz, Argentina). Relaciones de la Sociedad Argentina de Antropología VII:85-114.

CAVIGLIA, S. 1986. Nuevos restos de cánidos tempranos en sitios arqueológicos de Fuego-Patagonia. Anales del Instituto de la Patagonia 16:85-93.

DELLER, B. y C. ELLIS. 2001. Evidence for Late Paleo-Indian Ritual from the Caradoc Site (AfHj-104), Southwestern Ontario, Canada. American Antiquity 66(2):267284.

EMPERAIRE J., A. LAMING-EMPERAIRE y H. REICHLEN. 1963. La grotte Fell et autres sites de la region volcanique de la Patagonie chilenne. Journal de la Societé des Americanistes 52:167-254.

FELL, J. 1959. Inventario de los materiales de Cueva Fell, Museo Regional de Magallanes (Palacio Braun Menéndez), Punta Arenas. MS.

FLEGENHEIMER, N. 2003. Cerro El Sombrero: A Locality with a view. In: Where the South Winds Blow: Ancient Evidence of Paleo South Americans, editado por L. Miotti, M. Salemme y N. Flegenheimer, pp. 51-56. Center for the Study of the First Americans, Texas A\&M University, College Station.
FLEGENHEIMER N. y M. ZÁRATE. 1989. Paleoindian occupation at Cerro El Sombrero locality, Buenos Aires Province, Argentina. Current Research in the Pleistocene 4:12-13.

JACKSON, D. 2000. Los implementos de molienda en un campamento estacional del Holoceno Medio: implicancias funcionales y contextuales. Chungara Revista Chilena de Antropología Suplemento Especial 36 (1):95-103.

JACKSON, D. y C. MÉNDEZ. 2005. Primeras ocupaciones humanas en la costa del semiárido de Chile: patrón de asentamientos y subsistencia. En: Actas del XVI Congreso Nacional de Arqueología Chilena, pp. 493502. Tomé, 2003.

JACKSON, D., R. SEGUEL, P. BÁEZ y X. PRIETO. 1999. Asentamientos y evidencias culturales del Complejo Huentelauquén en la comuna de Los Vilos, Provincia del Choapa. Anales del Museo de Historia Natural de Valparaíso 24:5-28.

LLAGOSTERA, A. 1979. 9.700 years of maritime subsistence on the Pacific: An analysis by means of bioindicators in the North of Chile. American Antiquity 44 (2):309-324.

LLAGOSTERA A., R. WEISNER, G. CASTILLO, M. CERVELLINO y M. COSTA-JUNQUEIRA. 2000. El Complejo Huentelauquén bajo una perspectiva macroespacial y multidisciplinaria. En: Actas del XIV Congreso Nacional de Arqueología Chilena, Tomo I: 461-482. Copiapó.

MASSONE, M. 1981. Arqueología de la región volcánica de Pali Aike (Patagonia Meridional Chilena). Anales del Instituto de la Patagonia 12:95-124.

MASSONE, M. 1987. Los cazadores Paleoindios de Tres Arroyos (Tierra del Fuego). Anales del Instituto de la Patagonia 17:47-60.

MASSONE, M. 1996. Hombre temprano y paleoambiente en la región de Magallanes: Evaluación crítica y perspectivas. Anales del Instituto de la Patagonia 24:81-98.

MASSONE, M. 1999. Aproximación metodológica al estudio de las ocupaciones tempranas de cazadores terrestres en la región de Magallanes. En: Soplando en el viento... Actas de las III Jornadas de Arqueología de Patagonia, pp. 99-112. Universidad Nacional del Comahue e Instituto Nacional de Antropología y Pensamiento Latinoamericano, Neuquén-Buenos Aires.

MASSONE, M. 2004. Los cazadores después del Hielo. Colección Antropológica VII, Centro de Investigaciones Diego Barros Arana, Santiago.

MASSONE M. y A. PRIETO. 2004. Evaluación de la modalidad Cultural Fell 1 en Magallanes. Chungara Revista de Antropología Chilena Suplemento Especial 36(1):303315. 
MENA, F. 2000. Un panorama de la prehistoria de Aisén oriental: estado del conocimiento a fines del siglo. Serie Antropología de la Universidad San Sebastián 2:21-41.

MENA F., V. LUCERO, O. REYES, V. TREJO y H. VELÁSQUEZ. 2000. Cazadores tempranos y tardíos en la Cueva Baño Nuevo 1, margen occidental de la estepa centropatagónica (XI Región de Aisén, Chile). Anales del Instituto de la Patagonia 28:173-195.

MENA F. y O. REYES. 1998. Esqueletos humanos del Arcaico Temprano en el margen occidental de la estepa Centropatagónica (Cueva Baño Nuevo, XI Región). Boletín de la Sociedad Chilena de Arqueología 25:19-24.

MENA F. y O. REYES. 2001. Montículos y cuevas funerarias en Patagonia: Una visión desde Cueva Baño Nuevo 1, XI Región. Chungara Revista de Antropología Chilena 33 (1):21-30.

MENA F., O. REYES, T. STAFFORD y J. SOUTHON. 2003. Early human remains from Baño Nuevo 1 Cave, Central Patagonia Andes, Chile. Quaternary International 109-110:113-121.

MÉNDEZ, C., O. REYES y H. VELÁSQUEZ. 2007. Tecnología lítica en el alto río Cisnes (estepa extra andina de la XI Región de Aisén): primeros resultados. Boletín de la Sociedad Chilena de Arqueología, en prensa.

MENGHIN, O. 1952. Fundamentos cronológicos de la prehistoria de Patagonia. Runa 1:23-43.

MENGONI, G. 1980. Los materiales óseos de la cueva 2 de Los Toldos (Expedición Menghin), y una aproximación a la metodología de análisis de restos faunísticos. Runa XIII:59-68.

MIOTTI, L. 1996. Piedra Museo (Santa Cruz), nuevos datos para la ocupación Pleistocénica en Patagonia. En: Actas de las Segundas Jornadas de la Arqueología de la Patagonia: 27-38, Buenos Aires.

NAMI, H. 1987. Cueva del Medio: perspectivas arqueológicas para la Patagonia Austral. Anales del Instituto de la Patagonia 17:73-106.
PRIETO, A. 1991. Cazadores tempranos y tardíos en Cueva del Lago Sofía 1. Anales del Instituto de la Patagonia 20:75-99.

REYES, O. 2002. Funebria indígena en el curso inferior del valle del Río Ibáñez, margen occidental de la estepa de Centropatagonia (XI Región de Aisén). Anales del Instituto de la Patagonia 30:87-101.

STOTHERT, K. 1988. La prehistoria temprana de la península de Santa Elena, Ecuador; Cultura Las Vegas. Miscelánea Antropológica Ecuatoriana, Serie Monográfica 10, Museos del Banco Central del Ecuador, Quito.

STOTHERT, K y G. QUILTER. 1991. Archaic adaptation of the Andean region, 9000 to 5000 B.P. Revista de Arqueología Americana 4:25-53.

SUÁREZ, R. 2003. Paleoindian components of northern Uruguay: new data on early human occupations of the late Pleistocene and early Holocene. En: Where the South Winds Blow: Ancient Evidence of Paleo South Americans, editado por L. Miotti, M. Salemme y N. Flegenheimer, pp. 29-36. Center for the Study of the First Americans, Texas A\&M University, College Station.

SUÁREZ, R. y J. LÓPEZ. 2003. Archaeology of the Pleistocene-Holocene transition in Uruguay an overview. Quaternary International 109-110:65-76.

TADDEI, A. 1987. Algunos aspectos de la arqueología prehistórica del Uruguay. Estudios Atacameños 8:62-93.

TREJO, V. y D. JACKSON. 1998. Cánidos patagónicos: Identificación Taxonómica de mandíbulas y molares del sitio arqueológico Cueva Baño Nuevo 1 (Alto Nirehuao, XI Región). Anales del Instituto de la Patagonia 26:181-194.

VALVERDE, F. 2006. Estrategias de conservación y economía de la materia prima lítica en contextos tempranos de la Sierra de Tandilla Oriental. Cazadores-Recolectores del Cono Sur, Revista de Arqueología 1:179-187.

VELÁSQUEZ, H. y F. MENA. 2006. Distribuciones óseas de ungulados en la Cueva Baño Nuevo-1 (XI Región, Chile): un primer acercamiento. Magallania 34(2):91-106. 